\title{
IMPROVing EARNings PREDICTIONS With NEURAL NETWORK MODELS
}

\author{
RĂZVAN POPA*
}

\begin{abstract}
In this paper we develop a generalized deep neural network model to predict quarterly earnings. Using a diverse range of predictors consisting of fundamental, technical and sentiment data the resulting model outperforms existing timeseries models such as the Fama-French 2006 regression model and comes close in prediction accuracy to sales analysts' estimates. This is achieved by handling some known issues in time series models such as seasonality and non-linearity of the earnings while improving predictions with additional explanatory variables that reflect the expectations of the market. Thus, we add to the existing literature a comprehensive and innovative neural network model that provides solutions to known challenges in forecasting and closes the gap between statistical models and sales analysts.
\end{abstract}

Keywords: Comparative analysis, earnings forecasting methods, Fama French profitability model, deep neural network

\section{INTRODUCTION}

Whether it is academic researchers that require reliable predictions for future profitability in their research studies or investors that base their resource allocation on earnings expectations, the importance of accurate earnings forecasts cannot be overlooked. This challenge is mostly prevalent in the field of valuation (academic and professional) where profitability forecasting plays an important role in deciding the present value of a company based on its future cash flows.

The difficulty that lies in predicting earnings is closely related to their nature. First, earnings and profitability as a whole tend to be persistent (Fama and French 2006). This is related to the continuity and durability of the current earnings that allows companies to present quality earnings for long periods of time. Another two characteristics that are

\footnotetext{
* Alexandru Ioan Cuza University of Iasi, Faculty of Economics and Business Administration, Iași, Romania, e-mail: razvan.popa2010@gmail.com
} 
amplified at quarterly reporting frequency are that earnings are seasonal (Kang 1991, Hill, et al. 1994) and non-linear (Donaldson, Kamstra and Kim 1993). This means that earnings are difficult to predict from one quarter to another since seasons, consumer behavior and other macro-economic conditions produce variability. This variability is then translated into their non-linear nature which makes it difficult for linear models to fit a function between them.

Looking into existing solutions, two main groups of earnings predictions sources arise: time series models (TS) and sales analysts' (SA) estimates. These two groups closely go into a debate of which one is superior with certain studies showing that SA significantly outperform TS models forecasts (Brown and Rozeff 1978, Collins and Hopwood 1980, Fried and Givoly 1982, Wiedman 1996) while others state that SA estimates are not significantly more accurate than TS models (Cragg and Malkiel 1968, Elton and Gruber 1972, Imhoff and Pare 1982).

From an outside perspective certain advantages and disadvantages can be observed for both approaches. First, there is significant evidence that SA predictions tend to be biased upwards (O’Brien 1988, Mendenhall 1991, L. Brown 1993, Das, Levine and Sivarmakrishnan 1998) while overlooking information when forecasting earnings (Fama and French 2006). Second, TS models are prone to some short-comings when compared to SA when it comes to the timing advantage and contemporaneous advantage (Brown, Richardson and Schwager 1987). These translate into the time period from the last earnings report to the current one being predicted and a better utilization of the information on the date of the announcement.

Taking into consideration the short-comings of TS models we decided to add several explanatory variables to tackle the issue of the market expectations and timing advantage of the SA. Variables such as the news and Twitter sentiment taken one day before can provide an overview of the mood of the market and its expectations right before earnings. Another area that provides insight can be the options market where a ratio such as the put/call ratio can also show how bearish or bullish the market is at that time. Furthermore, information can also be found in peers or correlated companies that reported before the one in question.

To address the issues discussed above such as non-linear relations, seasonality but also model the great amount of data available we chose to tackle this problem using neural network models. Whether used for classification or regressions, neural network (NN) models have been around for quite a while, but it's in the last decade that it's been picking up steam due to hardware advancements and the financial field is no exception.

Specifically, predicting quarterly earnings using neural networks has been tried before with various degrees of success, thus the ongoing debate if NN models provide superior results to TS. One aspect that is brought into discussion a lot of the times is that NN models tend to be context sensitive (Chatfield 1993, Hill, et al. 1994, J.L., P.C.Y. and Y. 1996). This translates into the fact that NN models tend to behave differently from one company to another. One reason is that the amount of data required by NN models is quite large and earnings announcements come in relatively small amounts. We target this issue in our model by not making it company specific, rather a general model where all companies and earnings 
events are pooled together and analyzed as a whole. Thus, our model is built on the whole dataset available consisting of all companies and earnings events. In abstract terms, we can think of the developed NN model as a general formula for predicting earnings for any company that is part of the US stock market.

On the other hand, more recently we find studies which state that NN can better model the non-linear structure of the earnings than linear, time series models both for the univariate and multivariate case (Zhang, Cao and Schniederjans 2004). This is one of the main reasons we chose to proceed using NN models instead of existing regression-based models.

Considering the ongoing debates, we aimed to provide a comparative analysis between sales analysts, time series models and neural network models forecasts. Also, we add to the existing literature a generalized NN model with an expanded set of explanatory variables that go beyond the theoretical, financial information that we are used to in financial studies.

Starting with the analysts' estimations we chose the IBES (Institutional Brokers' Estimate System) data for its sufficient timeframe and consistency with existing research papers. These predictions come in the form of the mean of the SA estimates and usually rely on an average of 10 analysts.

Choosing a TS model for our study however required more thought as it needed to be multivariate and employ as much explanatory information as possible in order to be a fair comparison to the neural network model. For this reason, but also due to the large timespan between the moment of writing this paper and the release of those models we chose to not employ some estimation techniques used commonly in the existing literature such as Brown and Rozeff (1979), Foster (1977) or Griffin-Wats (1977). Instead, we selected a more comprehensive and recent model developed by Fama and French (2006), further referred to as FF06 model to forecast profitability using a set of financial ratios, stock prices, returns, a variable that denotes the probability of default (Ohlson 1980) and a composite measure of the firm's strength (Piotroski 2000). This model addresses a lot of the characteristics of earnings including persistence (Fama and French 1995), the prices and returns as leading indicators (Weiss, Naik and Tsai 2008) and accruals as a mean of earnings generation. One modification that we add to the model is predicting quarterly earnings instead of annual earnings as in the original paper, alteration that has been suggested before in literature (Harris, F., \& Wang, 2013).

For our model, we chose to employ one of the most recent fields in machine learning, mainly, deep learning. This type of learning structures algorithms into layers creating an artificial neural network that is able to learn and make decisions on its own. We took this decision to address the non-linearity, seasonality and complex structure of earnings and to simulate the way the sales analysts make predictions by replicating the structure of the human brain. An immediate debate that appears in literature is on the training algorithm used to train the neural network or how to help it learn from existing data. There are several studies that are in favor of using a genetic algorithm instead of the backward propagation algorithm. Backward propagation involves an iterative adjustment of a single parameter vector that has 
the objective to minimize a certain cost function. In a genetic algorithm a new generation of parameter vectors is developed by changing the parameter vectors in the current generation (Dorsey and Mayer 1995, Sexton, Dorsey and Johnson 1999). This technique should increase the probability of finding a global minimum by simultaneously exploring different parts of the parameter space (Dorsey and Mayer 1995). As previously stated, conflicting results appear in the existing literature with those that favor genetic algorithm (Cao and Parry 2009) when mean squared error is used as a cost function while for the same cost function and the mean absolute error backward propagation seems to behave better in other studies (Sarchami and Eftekhari 2012). In our model we decided to use the backward propagation algorithm since we have seen no signs of local optimum issues thanks to the efficiency of the Xavier initialization method used (Glorot and Bengio 2010). Also, the superior execution speed compared to the genetic algorithms played an important role in making this decision too.

\section{RESEARCH OBJECTIVE}

The purpose of this paper is to conduct research based on the expectation that the deep neural network model developed is superior to the FF06 model for profitability (in both cases: using the same variables and the added sentiment and correlation variables) and whether it is a reliable alternative to the SA estimates. Thus, we come up with the following hypotheses that we want to test:

H1. The DNN is superior to the linear regression when using the FF06 profitability model's explanatory variables.

H2. The DNN model is superior to the FF06 profitability model when using the added, developed explanatory variables.

H3. The DNN model is superior to the SA estimates when using the added, developed explanatory variables.

\section{METHODOLOGY}

\subsection{Fama French profitability model}

The Fama French model for profitability employs a diverse range of predictors to estimate profitability which is defined as earnings $(Y)$ divided by the book equity $(B)$. Some caveats inferred from existing literature on which the model is based include the following: profitability is persistent, current investment is related to future profitability, accruals forecast profitability, dividend paying firms tend to be more profitable and smaller firms tend to be more profitable. Thus, the following regression (equation 1) is estimated:

$$
Y_{t+1}=\operatorname{Int}+\beta_{1} \times \ln \left(B_{t} / M_{t}\right)+\beta_{2} \times \ln \left(M C_{t}\right)+\beta_{3} \times \operatorname{Neg} Y_{t}+\beta_{4} \times\left(Y_{t} /\right.
$$

$\left.B_{t}\right)+\beta_{5} \times\left(-A C_{t} / B_{t}\right)+\beta_{6} \times\left(+A C_{t} / B_{t}\right)+\beta_{7} \times\left(d A_{t} / A_{t}\right)+\beta_{8} \times N o D_{t}+\beta_{9} \times\left(D_{t} /\right.$

$\left.B_{t}\right)+\beta_{10} \times 1 Y_{r_{t}}+\beta_{11} \times 2-3 Y_{r_{t}}+\beta_{12} \times O H_{t}+\beta_{12} \times P T_{t}+\beta_{12} \times\left(I_{t} / B_{t}\right)(\mathbf{1})$

where,

$$
B_{t}=\text { book value per share }
$$




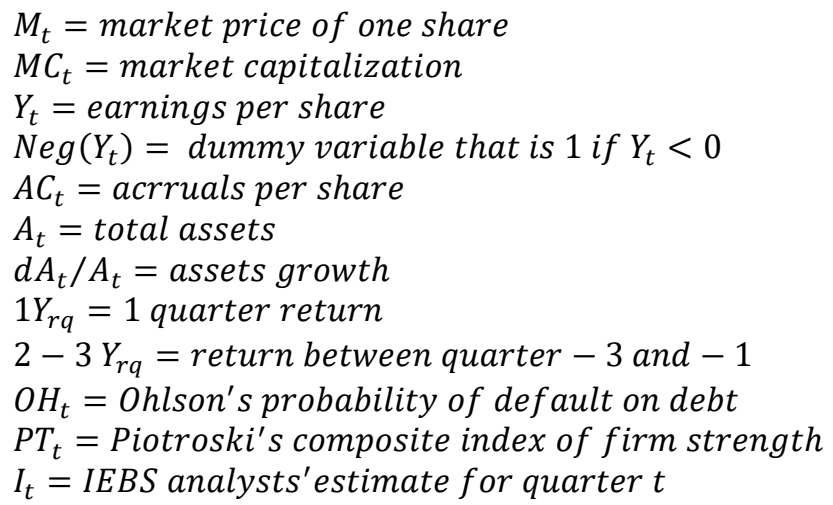

Valuation theory says that expected stock returns are related to three variables: the book-to-market equity ratio $\left(\mathrm{B}_{\mathrm{t}} / \mathrm{M}_{\mathrm{t}}\right)$, expected profitability, and expected investment. Given $\mathrm{B}_{\mathrm{t}} / \mathrm{M}_{\mathrm{t}}$ and expected profitability, higher expected rates of investment imply lower expected returns. But controlling for the other two variables, more profitable firms have higher expected returns, as do firms with higher $\mathrm{B}_{\mathrm{t}} / \mathrm{M}_{\mathrm{t}}$. These predictions are confirmed in our tests.

The data source used in our reproduction is the same data used in the original model: financial data from Compustat, Price data from CSRP and analyst estimates from IBES. We employ the same filtering method for the stocks as in the original paper: Stocks with total assets greater than 25 million USD and book equity greater than 12.5 million at each point in time. Companies with values outside the 0.5 and 99.5 percentile are also excluded. All variables used are per share.

To adapt the model for quarterly earnings forecasting and provide a fair comparison with the NN model and analysts' estimates we generalized as well instead of keeping it company specific. This is also an outcome of the fact that the amount of data remaining per company after filtering that contains sentiment information is reduced in size. Thus, we do one regression for all data instead of one regression for each company. Lastly, we remove the final explanatory variable from the model $\left(\mathrm{I}_{\mathrm{t}} / \mathrm{B}_{\mathrm{t}}\right)$ since we want to compare the models as if there was no analyst estimates available. Also, for an apple to apple comparison, the dependent/forecasted variable becomes earnings per share instead of earnings divided by book equity per share. This is done with the background knowledge that the explanatory power of the model remains the same since the removed variable $\left(\mathrm{B}_{\mathrm{t}}\right)$ is already present in the explanatory variables in multiple ratios used to scale the inputs for companies of different sizes.

The model is estimated using the Ordinary Least Squares (OLS) method on the $80 \%$ of the dataset (or the training dataset as referred later) pooling together all the companies. Thus, even though the model is not company specific anymore, the large amount of data provided allows for a comparable performance to the original model. 
Table 1: OLS estimation for the FF06 profitability model

\begin{tabular}{|c|c|}
\hline Variable & FF06 model for profitability \\
\hline Constant & $\begin{array}{l}-1.045^{* * *} \\
(0.034)\end{array}$ \\
\hline $\ln \left(\mathrm{B}_{\mathrm{t}} / \mathrm{M}_{\mathrm{t}}\right)$ & $\begin{array}{l}-0.312 * * * \\
(0.007)\end{array}$ \\
\hline $\ln \left(\mathrm{MC}_{\mathrm{t}}\right)$ & $\begin{array}{l}-0.023 * * * \\
(0.008)\end{array}$ \\
\hline $\operatorname{Neg}\left(Y_{t}\right)$ & $\begin{array}{l}-0.265^{* * *} \\
(0.016)\end{array}$ \\
\hline $\mathrm{Y}_{\mathrm{t}} / \mathrm{B}_{\mathrm{t}}$ & $\begin{array}{l}0.495 * * * \\
(0.028)\end{array}$ \\
\hline$-\mathrm{AC}_{\mathrm{t}} / \mathrm{B}_{\mathrm{t}}$ & $\begin{array}{l}-0.350 * * * \\
(0.041)\end{array}$ \\
\hline $\mathrm{AC}_{\mathrm{t}} / \mathrm{B}_{\mathrm{t}}$ & $\begin{array}{l}0.063 \\
(0.059)\end{array}$ \\
\hline $\mathrm{dA}_{\mathrm{t}} / \mathrm{A}$ & $\begin{array}{l}-0.036 \\
(0.039)\end{array}$ \\
\hline $\operatorname{No}\left(D_{t}\right)$ & $\begin{array}{l}-0.097 * * * \\
(0.010)\end{array}$ \\
\hline $\mathrm{D}_{\mathrm{t}} / \mathrm{B}_{\mathrm{t}}$ & $\begin{array}{l}-0.001 * * * \\
(0.000)\end{array}$ \\
\hline $1 \mathrm{Qr}_{\mathrm{t}}$ & $\begin{array}{l}-0.081 * * * \\
(0.018)\end{array}$ \\
\hline $2-3 \mathrm{Qr}_{\mathrm{t}}$ & $\begin{array}{l}-.054 * * * \\
(0.014)\end{array}$ \\
\hline $\mathrm{OH}_{\mathrm{t}}$ & $\begin{array}{l}-0.100 * * * \\
(0.003)\end{array}$ \\
\hline $\mathrm{PT}_{\mathrm{t}}$ & $\begin{array}{l}0.007 * * * \\
(0.003)\end{array}$ \\
\hline $\begin{array}{l}\text { Observations } \\
\text { Adjusted R-squared }\end{array}$ & $\begin{array}{l}16177 \\
0.442\end{array}$ \\
\hline
\end{tabular}

Standard errors in parentheses; $* * * \mathrm{p}<0.01$

We can immediately observe that the coefficients are in line with the original model, with an F-statistic that supports the significance of the explanatory variables. Looking at the coefficients we confirm the fact that the Ohlson's default profitability has a negative effect on earnings and the Piotroski's strength score adds to the overall earnings value (in a small amount though). Past profitability $\left(\mathrm{Y}_{t} / \mathrm{B}_{\mathrm{t}}\right)$ seems to carry the most amount of predictive power followed by the negative accruals and the book equity to price ratio.

\subsection{Deep neural network}

Neural networks were inspired from the studies on the information processing capabilities of the human brain. Some characteristics of the human brain that were replicated in the neural networks include its nonlinear and parallel structure and dense connections between neurons / nodes (figure 1). 


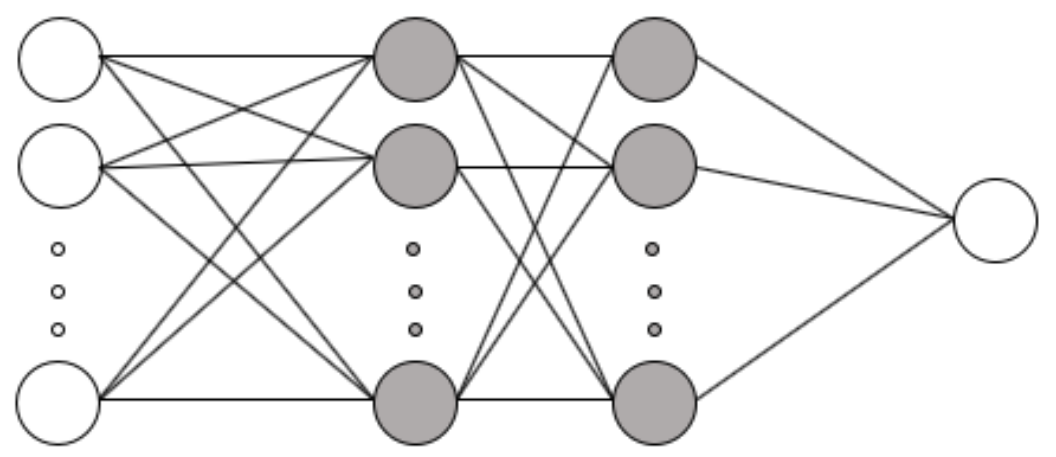

Figure 1: Schematic overview of a deep neural network with two hidden layers. The input layer (first layer) has $n$ neurons representing the $n$ explanatory variables used and the output layer (last layer) consists of one neuron representing the forecasted earnings per share. The layers in grey are the two hidden layers employed and each have m nodes.

Neural network $(\mathrm{NN})$ models reproduce the inner workings of the human brain by linking layers of input and output variables with processing units called hidden nodes. Our model employs an input layer with seventy nodes (the number of explanatory variables/features), two hidden layers with eight nodes each and an output layer consisting of one node (the earnings per share prediction).

Each explanatory variable (feature) has a weighted connection to each node in the first hidden layer, with the latter being connected in the same way to the second hidden layer and finally to the output node/layer.

The value of a neuron is calculated as a weighted sum of the input variables plus a bias (constant) for each input as in equation (2):

$$
Y=\sum(\text { weight } * \text { input })+\text { bias (2) }
$$

Similar to the brain, we must decide when these neurons fire (activate) or not. This is done via activation functions like the most basic one called the step activation. This activates the neuron when a certain value (threshold) is exceeded by Y.

One important aspect when setting up a neural network is how each weight is initialized. The way the weights are assigned initially can make the difference between a model that finds the global optimum and a model that gets stuck in a local one. One common practice is to initialize these weights randomly each time the model is trained.

The last aspect that has to be considered when working with neural networks is how the model learns. This is done via an algorithm called backpropagation which represents how the weights are adjusted after each training example. After an estimation is done on a training example the resulting error is calculated and the weights are adjusted from the output to the input accordingly. Using differential calculus, the instantaneous rate of change is calculated 
using partial derivatives for each parameter and the model is adjusted using a chain rule of calculus.

As mentioned earlier, we refer at the estimation step as the training and validation part. Training represents the data that is given as example to the model to learn and validation is the data used to check the behavior of the model. Both are chosen randomly each time the model is trained. The training procedure is repeated as long as the model continues to learn (improve its estimates) for a number of iterations, also known as epochs. After an acceptable model is achieved it is tested against a testing dataset which represents new data that the model has never seen before, such that a real-world performance indication can be provided.

\subsubsection{Implementation}

To develop and test our neural network model we chose to use the TensorFlow package due to its robustness, high performance $\mathrm{C}++$ implementation and Python specific usability. For productivity purposes we chose the high-level API built on top of it, Keras.

We chose Keras since we were aiming for a deep-neural-network (DNN) implementation that allowed us to model the complex non-linear relations between our large and diverse set of input variables. This architecture is represented by a feed forward neural network with multiple hidden layers capabilities where the information flows from the input to the output and adjusts the weights of each after each epoch.

\subsubsection{Methodology}

A first recommended step when training neural network models is to normalize the data. This is required as a great variability in the ranges of the data can make the gradient descent algorithm run slow (Ioffe and Szegedy 2015). We did this in two steps. First all financial data were transformed into per share data, in this way standardizing across all companies. Next, all variables were normalized using a Gaussian normalization method that replaces each value with its $\mathrm{z}$-score. (demeaned value divided by the standard deviation).

When setting up the model, one of the first decisions we have to make is how many nodes and hidden layers to use. In case of multi-layer neural networks, a belief is that in order for the model to achieve full generality a number of two hidden layers is necessary (Sontag 1992). We thus arrive at a configuration of 4 dense layers (one input, two hidden and one output).

Closely following after deciding on the number of layers is how many nodes per hidden layer to use. An indication that we considered when started training the model is that we need to specify as many hidden nodes as principal components needed to capture 70-90\% of the variance of the input data (Boger and Guterman 1997). In our case this proved to be around 6-14 nodes and after a few validations runs of the model we set our hidden layers at a number of 8 nodes.

For the activation function we went with a non-linear function, namely the leaky rectified linear unit (Maas, Hannun and $\mathrm{Ng}$ 2013) that increases the range of the classic 
rectified linear unit which is one the most used activation functions in deep neural networks and proved to be a good fit for the nonlinearity of our data.

We then chose our initialization function, which in combination with the number of epochs parameter allows our model to learn smoothly throughout time. For the initialization function we chose the Adadelta initialization (Zeiler 2012) which adapts the learning rates based on a moving window of gradient updates, instead of accumulating all past gradients. This allows it to continue learning even when many updates have been done. After running the model a few times, we arrived at a number of 300 epochs in combination with a learning rate of 0.1 that allowed our model to learn in a steady and improving way over time.

As a loss function we chose the mean absolute error (MAE) since other loss functions like mean square error (MSE) tends to not necessarily increase with the variance of the errors as the MAE does. Also, MAE bears the most meaning when comparing models.

\subsubsection{Feature selection}

To train the model we fed two types of variables: lagged financials and forwardlooking variables. The lagged financials included variables such as total assets, liabilities, cost of goods sold, sales and past earnings.

The forward-looking variables included: past-quarter next-day return and volume change to provide information regarding guidance for this quarter; previous day information like put/call open interest, news and twitter sentiment, average surprise produced by peers, price correlation weighted average of past earnings surprise of other companies and changes in price and volume in the last days before the announcement

We decided to use the past 4 quarters lagged variables to overcome seasonality effects and also include the earnings trend over quarters. The next step in building our model consisted in selecting only the features that added explanatory power to our model. For this task we used a procedure called Sequential Floating Backward Selection (Somol, Novovičová and Pudil 2010) which starts with an empty set of features and builds upon it as long as the objective function is increased for $\mathrm{k}$ variables.

1. Start with the empty set $Y=\{\varnothing\}$

2. Select the best feature

$$
\begin{aligned}
& x^{+}=\operatorname{argmax}\left[J\left(Y_{k}+x\right)\right] \\
& Y_{k}=Y_{k}+x^{+} ; \quad k=k+1 ; \\
& \text { 3. Select the worst feature } \\
& x^{-}=\operatorname{argmax}\left[J\left(Y_{k}-x\right)\right] \\
& \text { 4. If } J\left(Y_{k}-x^{-}\right)>J\left(Y_{k}\right) \text { then } \\
& Y_{k+1}=Y_{k}-x ; \quad k=k+1 ; \\
& \text { go to step } 3 \\
& \text { else } \\
& \text { go to step } 4
\end{aligned}
$$

Algorithm 1: Pseudocode representation of the variable selection algorithm (BFBS).

\subsubsection{Validation}


With the DNN model set up we train the model and validate it for a number of 300 epochs (learning cycles) where at each iteration the learning and validation sets are randomly created. In figure 2 we observe a correct behavior of the gradient descent algorithm that descends fast into an acceptable solution in a few dozen iterations and then slowly decreases to the global optimum. As expected, the validation error is slightly higher than the training error which suggests no signs of overfitting or underfitting.

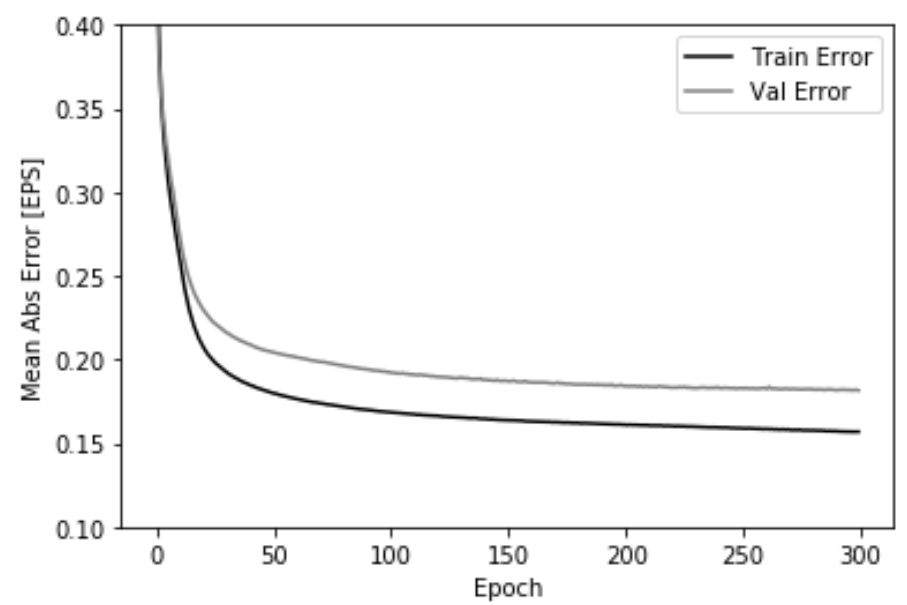

Figure 2: DNN model training and validation error across epochs

\subsection{Comparison of methods}

All models are run and compared on the testing dataset, which, in our view represents the future, unknown earnings events, thus proving a good proxy for how the models would behave in a real-world application. Throughout the testing procedure we employed three metrics: mean absolute error (MAE), mean absolute percentage error and mean squared error (MSE). Also, R-squared is used to show the explanatory power of the models, again on the testing dataset.

\section{Data}

The financial instruments used throughout this paper are North American, US listed companies that are part of the Russel3000 Index. We choose this index for its broad spread, financial data availability and overall consistency reasons.

There are 25 fundamental variables and one technical as required by the Fama French profitability model. For the neural network model another 7 variables are fetched/derived: Twitter and News Sentiment data, Put/Call Open Interest, Price and Volume (with lags), average subsector surprise and composite earnings score of price correlated companies. Other variables are present with identification/descriptive roles. We begin with data within the timeframe 1979-2019, with most of it laying in the 2013-2019 area after filtering the entries that do not have sentiment and options data. 


\subsection{Data collection}

The index constituents alongside with their sector and sub-sector are fetched using Bloomberg and then filtered to match the requirements of the Fama French model for profitability (No companies from the Financial or Real Estate Sectors). This results in a total of 2124 stocks. The data used comes from two main providers and five different sources with a timeframe between 1992 and 2019 (table 2).

Table 2: Data providers and sources used

\begin{tabular}{ccc} 
Provider & Source & Description \\
\hline $\begin{array}{c}\text { Wharton Research } \\
\text { Data Services }\end{array}$ & $\begin{array}{c}\text { The Center for Research } \\
\text { in Security Prices } \\
\text { (CRSP) }\end{array}$ & $\begin{array}{c}\text { Daily Stock File: Price, } \\
\text { Volume and Share Factor }\end{array}$ \\
\hline $\begin{array}{c}\text { Wharton Research } \\
\text { Data Services }\end{array}$ & $\begin{array}{c}\text { Compustat-North } \\
\text { America Daily - } \\
\text { Fundamentals Quarterly }\end{array}$ & $\begin{array}{c}\text { The fundamental data that } \\
\text { is used throughout this } \\
\text { paper }\end{array}$ \\
\hline $\begin{array}{c}\text { Wharton Research } \\
\text { Data Services }\end{array}$ & $\begin{array}{c}\text { IBES from Thomson } \\
\text { Reuters - Summary } \\
\text { History - Surprise } \\
\text { History }\end{array}$ & $\begin{array}{c}\text { EPS actual value, } \\
\text { estimate and } \\
\text { announcement date }\end{array}$ \\
\hline Bloomberg & Historical & $\begin{array}{c}\text { News Sentiment, Twitter } \\
\text { Sentiment, Put/Call Open } \\
\text { Interest }\end{array}$ \\
\hline Bloomberg & Bulk Historical & $\begin{array}{c}\text { Earnings Announcement } \\
\text { Time (before/after the } \\
\text { bell) }\end{array}$ \\
\hline
\end{tabular}

\subsection{Data processing}

The data are processed and merged using the CUSIP codes and tickers alongside the announcement dates and their corresponding financial quarters. Then the lagged prices, volumes and sentiment data are added using the announcement date and announcement time to derive which is the last trading day before the earnings report. For each company we look into its peers (sub-industry) and compute the average surprise produced by the ones who reported before it. For the composite earnings score based on price correlation a correlation weighted average of the earnings surprise is computed from the companies that reported before the current announcement date.

\subsection{Data filtering}

An initial prefilter is made to ensure that all mandatory variables required by the Fama French model are present and not null with the missing optional variables set to 0 . As required by the profitability model, we ensure that each company has total assets greater than 25 million USD at each point in time. 
Next, we ensure that we provide the required number of lagged quarters (4) and split the data chronologically (80/20) into estimation and testing. The estimation is split again randomly this time into training and validation for the neural network model.

All data gathering, processing and filtering is done automatically with the help of several Python scripts/modules and apis from the data providers.

\subsection{Data structuring}

After the data have been gathered, processed and filtered, they are stored in a Python, Pandas Dataframe. This data structure is similar to a table where each row represents a separate input for our models. A row contains the current data (after the announcement date) that need to be forecasted, as well as the four preceding quarters. Using this type of structure, we encapsulate in one row all the current and historical information a model needs to forecast earnings.

\section{RESULTS}

Looking at earnings per share we immediately observe a skewness to the right which can be explained by the fact that highly unprofitable firms are not that common and usually result in bankruptcy as show by Ohlson (1980).

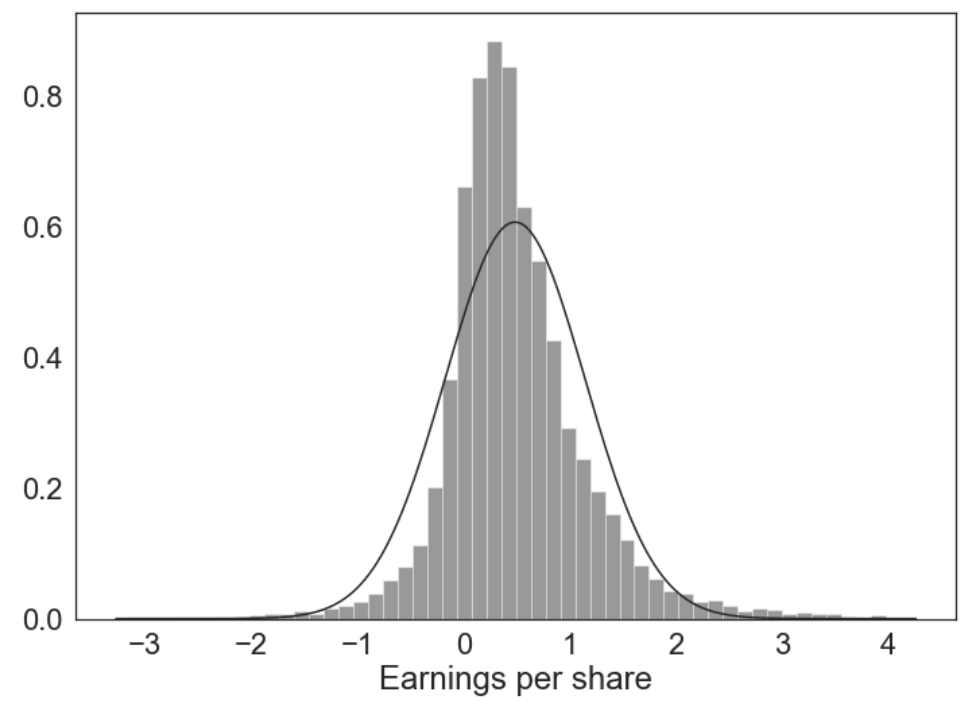

Figure 3: Earnings per share in US dollars distribution

Looking at the earnings surprise generated by the SA estimates we can observe the bias discussed in literature where SA tend to overestimate results thus resulting in an earnings surprise skewed to the left. 


\section{SA Earnings Surprise}

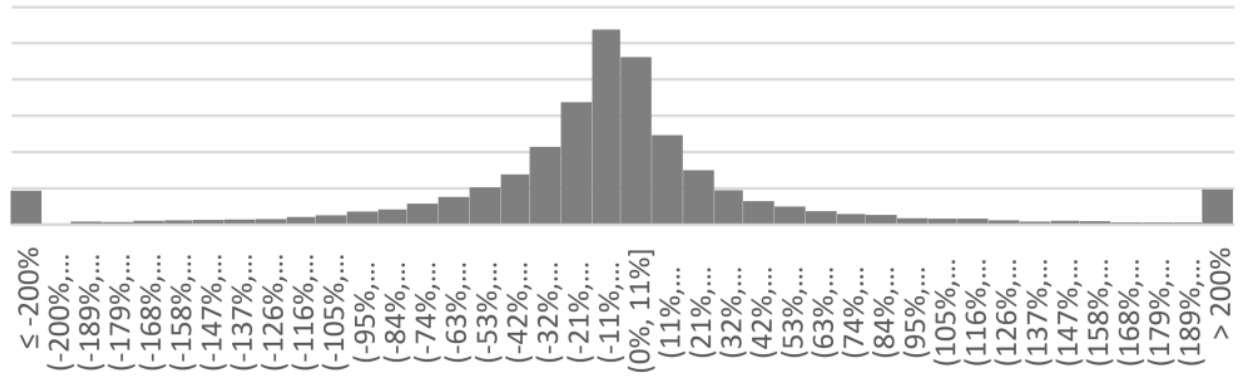

Figure 4: IEBS earnings surprise distribution calculated as (estimate/actual)-1

Looking into the explanatory power of past earnings we clearly see that a clear relationship can be observed, thus confirming the FF06 statement that past profitability forecast current profitability.

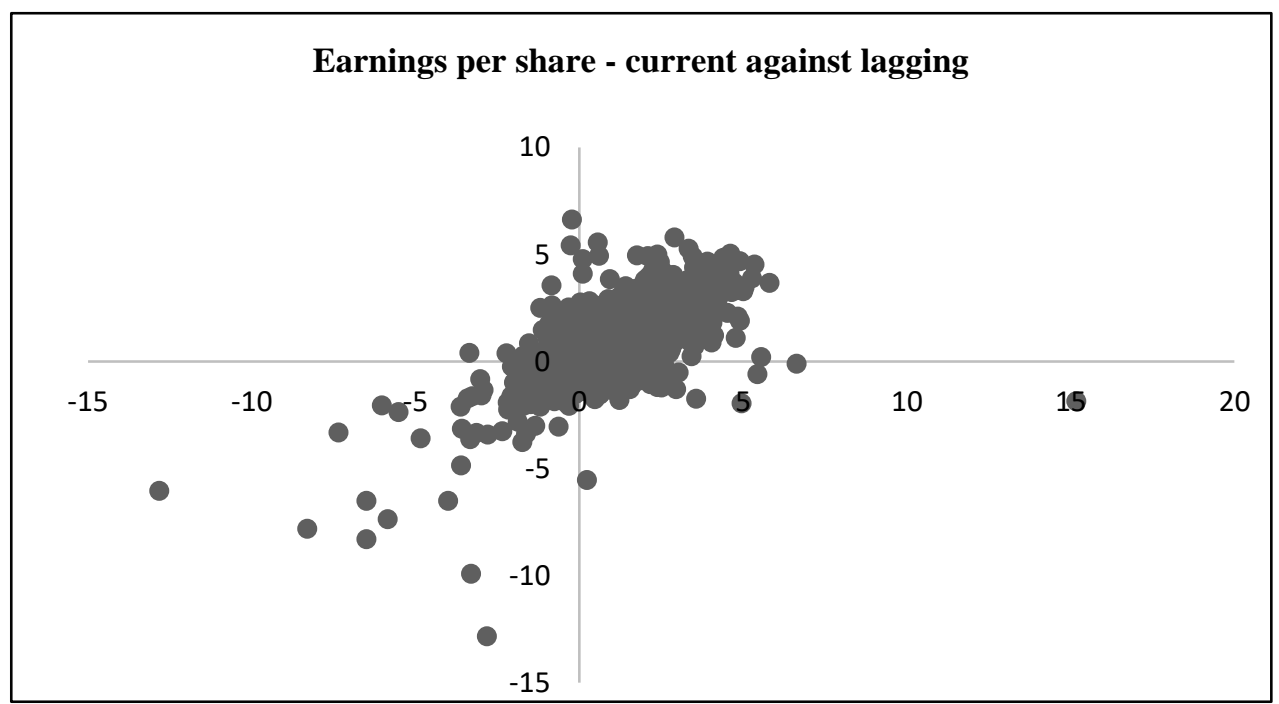

Figure 5: Current earnings per share (in US dollars) plotted against earnings per share one quarter before 


\section{REFERENCES}

Boger, Z., and H. Guterman. 1997. "Knowledge extraction from artificial neural network models." IEEE Systems, Man, and Cybernetics Conference. Orlando.

Brown, L. 1993. "Earnings forecasting research: It's implications for capital markets research." International Journal of Forecasting 9: 295-320.

Brown, L., G. Richardson, and S. Schwager. 1987. "An information interpretation of financial analyst superiority in forecasting earnings." Journal of Accounting Research 25: 49-67.

Brown, L.D., and M. Rozeff. 1979. "Univariate time series models of quarterly accounting earnings per share: A proposed model." Journal of Accounting Research 17: Journal of Accounting Research.

Brown, L.D., and M.S. Rozeff. 1978. "The superiority of analyst forecasts as measures of expectations." Journal of Finance 33: 1-16.

Cao, Qing, and Mark E. Parry. 2009. "Neural network earnings per share forecasting models: A comparison of backward propagation and the genetic algorithm." Decision Support Systems 47: 3241.

Chatfield, C. 1993. "Neural network: Forecasting breakthrough or passing fad." International Journal of forecasting 9: 1-3.

Collins, W.A., and W.S. Hopwood. 1980. "A multivariate analysis of annual earnings generated from quarterly forecasts of financial analysts and univariate time-series models." Accounting Research 18: 390-406.

Cragg, J.G., and B.C. Malkiel. 1968. "The consensus and accuracy of some predictions of the growth of corporate earnings." Journal of Finance 23: 67-84.

Das, S., B. Levine, and K. Sivarmakrishnan. 1998. "Earnings Predictability and Bias in Analysts' Earnings Forecasts." The Accounting Review 73: 277-94.

Donaldson, R.G., M. Kamstra, and H.Y. Kim. 1993. "Evaluating alternative models for conditional stock volatility". Working Paper, Vancouver: University of British Columbia.

Dorsey, R.E., and W.J. Mayer. 1995. "Genetic algorithms for estimation problems with multiple optima, nondifferentiability, and other irregular features." Journal of Business \& Economic Statistics 13 (3): 53-66.

Elton, E.J., and M.J. Gruber. 1972. "Earnings estimates and the accuracy of expectational data." Management Science 18: 409-424.

Fama, E.F, and K.R. French. 2006. "Profitability, Investment and Average Returns." Journal of Financial Economics 82: 491-518.

Fama, E.F., and K.R. French. 1995. "Size and book-to-market factors in earnings and returns." Journal of Finance 50: 131-155.

Foster, G. 1977. "Quarterly accounting data: Time series properties and predictive ability results." The accounting Review 52: 1-21.

Fried, D., and D. Givoly. 1982. "Financial analysts' forecasts of earnings: A better surrogate for market expectations." Journal of Accounting and Economics 4: 85-107.

Glorot, Xavier, and Yoshua Bengio. 2010. "Understanding the difficulty of training deep feedforward neural networks." Proceedings of the Thirteenth International Conference on Artificial Intelligence and Statistics. 249-256.

Griffin, P. 1977. "The time series behavior of quarterly earnings: Preliminary evidence." Journal of Accounting Research 15: 71-83.

Harris, Richard D. F., and Pengguo Wang. 2013. "An Improved Earnings Forecasting Model." SSRN. April. Accessed 03 20, 2019. https://ssrn.com/abstract=2802019.

Hill, T., L. Marquez, M. O'Conner, and W. Remus. 1994. "Artificial neural network models for forecasting and decision making." International Journal of Forecasting 10: 5-15. 
Imhoff, E.A., Jr., and P.V. Pare. 1982. "Analysis and comparison of earnings forecast agents." Journal of Accounting Research 20: 429-439.

Ioffe, Sergey, and Christian Szegedy. 2015. "Batch Normalization: Accelerating Deep Network Training by Reducing Internal Covariate Shift." International Conference on International Conference on Machine Learning. Lille. 448-456.

J.L., Callen, Kwan P.C.Y., and Yuan Y. 1996. "Neural Network Forecasting of Quarterly Accounting Earnings." International Journal of Forecasting 2 (14): 475-482.

Kang, S. 1991. "An investigation of the use of feedforward neural networks for forecasting".

Ph.D. Dissertation, Kent: Kent State University.

Maas, Andrew L., Awni Y. Hannun, and Andrew Y. Ng. 2013. "Rectifier Nonlinearities

Improve Neural Network Acoustic Model." International Conference on Machine Learning. Atlanta.

Mendenhall, R. 1991. "Evidence on the Possible Underweighting of Earnings Information." Journal of Accounting Research 29: 170-9.

O’Brien, P. 1988. "Analysts' Forecasts as Earnings Recommendations." Accounting \& Economics 10: 53-83.

Ohlson, J.A. 1980. "Financial ratios and the probabilistic prediction of bankruptcy." Journal of Accounting Research 18: 109-131.

Piotroski, J.D. 2000. "Value investing: the use of historical financial statement information to separate winners from losers." Journal of Accounting Research 38 (Supplement): 1-42.

Sarchami, Mohammad, and Mehdi Eftekhari. 2012. "Using artificial neural networks for forecasting per share earnings." African Journal of Business Management 6 (11): 4288-4294.

Sexton, R.S., R.E. Dorsey, and J.D. Johnson. 1999. "Optimization of neural networks: a comparative analysis of the genetic algorithm and simulated annealing." European Journal of Operational Research 114 (3): 589-601.

Somol, Petr, Jana Novovičová, and Pavel Pudil. 2010. "IMPROVING SEQUENTIAL

FEATURE SELECTION METHODS'." International Conference on Advances in Computer Science and Engineering. 689-001.

Sontag, E.D. 1992. "Feedback stabilization using two-hidden-layer nets." IEEE Transactions on Neural Networks 3: 981-990.

Weiss, D., P.A. Naik, and C.L. Tsai. 2008. "Extracting Forward-looking Information from Security Price: A New Approach." The Accounting Review 83: 1101-1124.

Wiedman, C. 1996. "The Relevance of Characteristics of the Information Environment in the Selection of a Proxy for the Market's Expectations for Earnings: "An Extension of Brown, Richardson, and Schwager [1987]."." Journal of Accounting Research 34 (2): 313-324.

Zeiler, Matthew D. 2012. ADADELTA: An Adaptive Learning Rate Method. Accessed 0320 , 2019. https://arxiv.org/pdf/1212.5701.pdf.

Zhang, W., Q. Cao, and M. Schniederjans. 2004. "Neural network earnings per share forecasting models: a comparative analysis of alternative methods." Decision Sciences 35 (2): 205237. 
Appendix A. DNN build procedure in Python

1. def build_model():

2. model $=$ keras.Sequential $([$

3. layers.Dense(8,

4. $\quad$ activation $=$ tf.nn.leaky_relu,

5. $\quad$ input_shape $=[$ len $($ estimation_dataset.keys ()$)]$

6. )

7. layers.Dense $(8$, activation $=\mathrm{tf} . n n$. leaky_relu $)$,

8. layers.Dense(1)

9. ])

10.

11. optimizer $=\mathrm{tf}$. keras.optimizers.Adadelta $(\mathrm{lr}=0.1)$

12.

13. model.compile(loss = 'mean_squared_error',

14. optimizer $=$ optimizer,

15. metrics $=$ ['mean_absolute_error', 'mean_squared_error'])

16. return model

Appendix B. Data filtering procedure in Python
1. FF_REQUIRED_COLUMNS = [
2.
'actq', \# Current Assets - Total
3.
'atq', \# Assets - Total
4.
'cheq', \# Cash and Short-Term Investments
'cogsq', \# Cost of Goods Sold
6.
'cshoq', \# Common Shares Outstanding
7.
'dlcq', \# Debt In Current Liabilities
8.
'dlttq', \# Long-Term Debt Total
9.
'dpq', \# Depreciation and Amortization
10.
'ibq', \# Income Before Extraordinary Items
11.
'lctq', \# Current Liabilities - Total
12.
'niq', \# Net Income (Loss)
13.
'saleq', \# Sales/Turnover (Net)
14.
'dvpsxq', \# Dividends per Share/Ex-Date
15.
'Itq', \# Liabilities - Total
16.
'scfq', \# Cash Flow Format
17.
'oancfy', \# Operating Activities/Net Cash Flow
18. ]
19.
20. FF_OPTIONAL_COLUMNS = [
21. 'dd1q', \# Long-Term Debt Due In One Year
22. 'pstkq', \# Preferred/Preference Stock (Capital) - Total
23.
24.
25.
'txdiq', \# Income Taxes - Deferred
'txditcq', \# Deferred Taxes and Investment Tax Credit
'wcapq', \# Working Capital (Balance Sheet) 
27. 'sstky', \# Sale of Common and Preferred Stock

28.

29. NN_COLUMNS = [

30. 'cshtrq', \# Common Shares Traded - Quarterly

31. 'prccq', \# Price Close-Quarterly

32. 'prchq', \# Price High - Quarterly

33. 'prclq', \# Price Low - Quarterly

34. ]

35.

36.

37. def ff_filter(df):

38. df.drop(df.columns[[0, 1, 2, 3, 4, 5, 6, 7, 10, 11, 13, 35]], axis $=1$, inplace=True)

39.

40. df.dropna(subset=FF_REQUIRED_COLUMNS, inplace=True)

41.

42. for optional_column in FF_OPTIONAL_COLUMNS:

43. df[optional_column] $=\mathrm{df}[$ optional_column].fillna $(0)$

44.

45. book_equity $=[]$

46. for $\mathrm{i}$, row in df.iterrows():

47. book_equity.append(row['atq'] - row['ltq'] + row['txditcq'] - row['pstkq'])

48.

49. df['book_equity'] = book_equity

50.

51. $\mathrm{df}=\mathrm{df}[\mathrm{df}[$ 'book_equity'] $>=12.5]$ \# Remove companies with less that $12.5 \mathrm{mln}$ in book equity in year

52.

53. $\mathrm{df}=\mathrm{df}[\mathrm{df}[$ 'atq'] $>=25] \quad$ \# Remove companies with less that $25 \mathrm{mln}$ in total ass ets in year $t$

54.

55. return $\mathrm{df}$

56.

57.

58. def eps_filter(df):

59. df.drop(columns=['TICKER', 'MEASURE', 'FISCALP'], axis=1, inplace=True)

60.

61. df.dropna(subset=['actual', 'surpmean'], inplace=True)

62.

63. df['anndats'] $=$ pd.to_datetime(df['anndats'], format='\%Y\%m\%d')

64.

65. dates $=[]$

66. quarters $=[]$

67. for $i$ in range(len(df)):

68. pyear $=$ df.iloc[i]['PYEAR']

69. pmonth $=$ df.iloc[i]['PMON'] 
70.

71. quarter $=\operatorname{int}(($ pmonth -1$) / 3)+1$

72.

73. dates.append('\%dQ\%d' \% (pyear, quarter))

74. quarters.append(quarter)

75.

76. $\mathrm{df}[$ 'datacqtr'] $=$ dates

77. df['quarter'] = quarters

78.

79. df.drop (columns=['PYEAR', 'PMON'], axis=1, inplace=True)

80.

81. df.rename (index $=$ str, columns $=\{$ 'OFTIC': 'tic' $\}$, inplace $=$ True $)$

82.

83. return $\mathrm{df}$

84.

85.

86. def technical_filter(df_t, df):

87. df_t.drop(columns=['PERMNO', 'COMNAM', 'CUSIP'], inplace=True)

88.

89. df_t['date'] = pd.to_datetime(df_t['date'], format='\% Y\% $\%$ \%')

90.

91. new_data $=\{\}$

92. for $i$ in range $(-5,1)$ :

93. new_data['volume $\%$ s' \% (str(i) if i != 0 else ")] = []

94. new_data['price\%s' \% (str(i) if i != 0 else ")] = []

95.

96. df $=$ df_t.reset_index ()$\cdot \operatorname{merge}(\mathrm{df}$,

97. how="left",

98. left_on=['TICKER', 'date'],

99.

100. right_on=['tic', 'anndats']

101.

102.

103.

104.

105.

106.

107.

108.

109.

110.

111.

112.

113. ).set_index('index')

114.

115 .

116.

df.dropna(subset=['anndats'], inplace $=$ True $)$

for $\mathrm{j}$ in range(len(df)):

if $\mathrm{j} \% 1000==0$ :

print('Step: \%d' \% j)

rt_adj = 1 if df.iloc[j]['report_time'] == 'after' else 0

index $=\mathrm{df}$.index $[\mathrm{j}]$

for $i$ in range $(-5,1)$ : new_data['volume\%s' \% (str(i) if i != 0 else ")] .append(df_t.iloc[index $+\mathrm{i}+\mathrm{rt} \_$adj]['VOL']) new_data['price\%s' \% (str(i) if i != 0 else ")] .append(df_t.iloc[index $+\mathrm{i}+\mathrm{rt} \_$adj]['PRC']) 
117.

118.

119.

for key in new_data:

120.

121.

df $[$ key $]=$ new_data[key $]$

122.

df.drop(columns=['VOL', 'PRC', 'date', 'TICKER'], inplace=True)

df.dropna(subset=['price', 'volume', 'price-1', 'volume-

$1 ']$, inplace $=$ True)

123.

124.

df.sort_values(by=['tic', 'anndats'], inplace=True)

125.

126.

127.

df.reset_index(inplace $=$ True $)$

128.

df.drop $($ columns $=[$ 'index'], inplace $=$ True $)$

129.

130.

return $\mathrm{df}$

131.

132.

133.

134.

def nearest(items, pivot):

return $\min ($ items, $k e y=$ lambda $\mathrm{x}$ : $\operatorname{abs}(\mathrm{x}-\mathrm{pivot}))$

135.

136.

137.

138.

139.

140.

141.

142.

143.

144.

145.

146.

147.

148.

149.

150.

151.

152.

153.

154.

155.

156.

157.

158.

159.

160.

161.

162.

def main():

\# Import fundamental data

df_f $=$ pd.read_csv(WRDS_FUNDAMENTAL_DATA_PATH)

df_f $=$ ff_filter(df_f $)$

\# Import eps values and estimates

df_e = pd.read_csv(WRDS_EPS_DATA_PATH $)$

df_tc $=$ pd.read_csv(WRDS_TICKER_CUSIP $)$

tickers $=[]$

for cusip in sorted(df_f['cusip'].unique().tolist()):

rows $=$ df_tc[df_tc['CUSIP'] $==$ cusip $[: 8]]$

if len(rows) $>0$ :

tickers.append(rows.iloc[0]['TICKER'])

df_e $=$ df_e[df_e['TICKER'].isin(tickers)]

df_e = eps_filter(df_e $)$

df $=$ df_f.merge(df_e, on=['tic', 'datacqtr'])

\# Import earnings report times

d_r $=$ pd.read_pickle(BMBG_BEFORE_AFTER_MKT_PATH) 
163.

164.

165.

166.

167.

168.

169.

170.

171.

172 .

173.

174.

175.

176.

177.

178. $\mathrm{y}=$ False)

179.

180.

181.

182.

183.

184.

185.

$$
\text { report_time }=[]
$$

for $i$ in range(len(df)):

bloomberg_ticker $=$ df.iloc[i]['tic'] + ' US Equity'

anndats $=$ df.iloc[i]['anndats']

closest_date $=$ nearest $\left(\mathrm{d} \_r[\right.$ bloomberg_ticker $] . \operatorname{keys}()$, anndats $)$

report_time.append(d_r[bloomberg_ticker][closest_date])

df['report_time'] = report_time

pd.to_pickle(df, 'wrds_partial.pkl')

\# Import technical data

df_t = pd.read_csv(WRDS_TECHNICAL_DATA_PATH, low_memor

$\mathrm{df}=$ technical_filter(df_t, df)

pd.to_pickle(df, MERGED_DATA_PATH)

if __name__ == '__ main__':

main() 